\title{
Electrolytes Change the Interfacial Water Structure but Not the Vibrational Dynamics
}

\author{
Malte Deiseroth, ${ }^{\dagger}$ Mischa Bonn, $^{\dagger}$ (๑) and Ellen H. G. Backus ${ }^{*}, \dagger \oplus$ \\ ${ }^{\dagger}$ Max Planck Institute for Polymer Research, Ackermannweg 10, 55128 Mainz, Germany \\ ${ }^{\ddagger}$ Department of Physical Chemisty, University of Vienna, Währinger Straße 42, 1090 Wien, Austria
}

\section{Supporting Information}

ABSTRACT: Heterogeneous ozone chemistry occurring on aerosols is driven by interfacial chemistry and thus affected by the surface state of aerosol particles. Therefore, the effect of electrolytes on the structure of interfacial water has been under intensive investigation. However, consequences for energy dissipation rates and mechanisms at the interface are largely unknown. Using time-resolved sum frequency generation spectroscopy, we reveal that the relaxation pathway is the same for neat water-air as for aqueous solutions of $\mathrm{Na}_{2} \mathrm{SO}_{4}$ and $\mathrm{Na}_{2} \mathrm{CO}_{3}$. We further show that similar lifetimes are extracted from all investigated systems and that these lifetimes show an excitation frequency dependent relaxation time from $0.2 \mathrm{ps}$ up to 1 ps. Hence, despite static SFG on the same systems revealing that
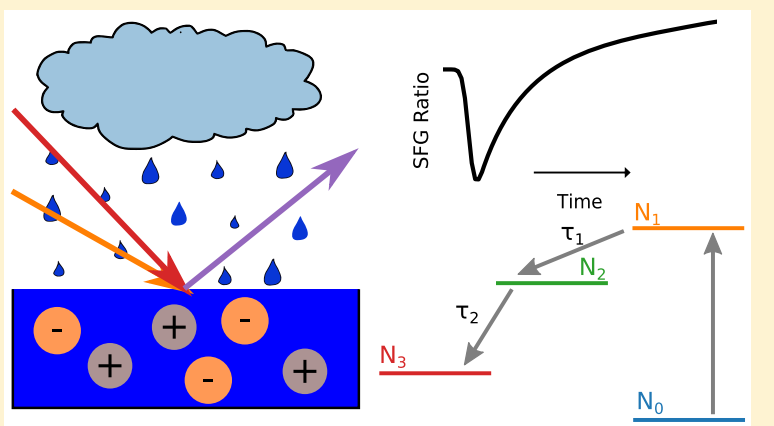
the interfacial aqueous structure changes upon adding electrolytes, the vibrational dynamics are indistinguishable for both pure water and different electrolyte solutions.

\section{INTRODUCTION}

Physical and chemical processes occurring at aqueous interfaces have been shown to play a prominent role in a variety of fields ranging from the chemistry of atmospheric aerosols and heterogeneous catalysis to biophysics and biochemistry. For instance, the heterogeneous ozone chemistry occurring on aerosols is largely driven by interfacial chemistry. ${ }^{1}$ In the case of atmospheric aerosols, the situation is especially complicated because of the large number of different components aerosols can contain. ${ }^{2}$ Among these components is a variety of ions, e.g., sodium, sulfate, and carbonate, originating from natural, as well as artificial, sources. ${ }^{3,4}$ Sulfate, for example, can be found in large quantities in urban areas, since it is released during industrial production processes. ${ }^{5} \mathrm{On}$ the other hand, roughly $30 \%$ of the $\mathrm{CO}_{2}$ released by human activity in the past few decades has been uptaken by the ocean and a large part of it now resides in the form of $\mathrm{CO}_{3}{ }^{2-}$ within the ocean itself. 6

Given its relevance to environmental chemistry, the structure of interfacial water in contact with ions has been under intensive investigation throughout the past decades. ${ }^{7-13}$ While knowledge of the structure of interfacial water is essential for understanding heterogeneous ozone chemistry, understanding the dynamics of these interfaces is also important, as in the course of chemical conversion on the surface of the aerosol transport of excess energy to and from the reaction sites is crucial. Yet, a molecular model of these transport mechanisms is currently missing. How is the energy transported to and from reaction sites? What is the effect of different ions on the transport mechanisms?
Here we study, using sum-frequency-generation (SFG) and its phase-resolved (PS) extension PS-SFG, the effect of ions on the structure and the vibrational dynamics of interfacial water. SFG is a second-order nonlinear process that is interfacespecific owing to its selection rules. ${ }^{14}$ We use an infrared (IR) beam in resonance with the $\mathrm{O}-\mathrm{H}$ stretch vibration of the water molecules. The $\mathrm{O}-\mathrm{H}$ stretch vibrational frequency is a marker of the strength of the local hydrogen bond network and the intensity provides information about the degree of alignment. $^{15}$

SFG and PS-SFG have already been used to study the effect of ions on the hydrogen bond network of interfacial water. It was found that ions can perturb the strength of the hydrogen bonds at the interface, even though the ions are located several hydration layers away from the interface. The results indicate that ions increase the strength of the hydrogen bond network. Furthermore, the relative order of ions at the interface was investigated and an ion distribution model was developed. With the help of PS-SFG the orientation of the dipole moment at the aqueous-air interface was studied and it was shown that certain ions can reverse the order of the dipole moment at the interfacial region of water. ${ }^{7-9}$

To study the vibrational energy flow, we present the first study on the vibrational dynamics of the water stretch vibration under the influence of ions. We use pump-probe (PP) SFG to probe the vibrational dynamics at the interface, the interface-

Received: August 28, 2019

Revised: September 11, 2019

Published: September 12, 2019 
specific counterpart to pump-probe IR spectroscopy. PP-SFG adds an additional pump laser that is tunable in frequency and time to the SFG setup. With its subpicosecond time resolution, it is well suited to measure the lifetimes of the water stretch vibration. PP-SFG can be used to measure how fast the involved oscillators lose their excitation energy, how vibrational energy is exchanged between molecules and how fast they change their hydrogen bond partners. Previous PP, IR, and SFG studies on water have shown a strong pump frequency dependence of the relaxation time. Weakly bonded hydrogen molecules relax slower than stronger bonded hydrogen molecules. ${ }^{16}$ Studies of water in contact with lipids at negative charged surfactants have revealed interesting coupling dynamics between different water species. ${ }^{17}$

As water in nature is rarely free of ions, it is important to understand the effects of ions on the water-vapor interface. It remains unclear whether the ion induced changes to the hydrogen bond network also influence the vibrational lifetimes of the involved molecular vibrations.

\section{METHODS}

Laser pulses are produced by an oscillator (Mai Tai, Spectra Physics) with a central wavelength of $800 \mathrm{~nm}$ and roughly 50 $\mathrm{nm}$ bandwidth. The Spitfire Ace is pumped by two Empowers $(22 \mathrm{~mJ}$ pulse energy at $527 \mathrm{~nm}$ ) and has a power output of about $10 \mathrm{~W}$ and a pulse length of $40 \mathrm{fs}$ with a repetition rate of $1 \mathrm{kHz}$. The sum frequency signal is generated using a focused broadband IR beam generated by a Topas-c with DFG stage (p-polarized, $15 \mu \mathrm{J}, 350 \mathrm{~cm}^{-1}$, centered at $2500 \mathrm{~cm}^{-1}$, angle $40^{\circ}$ to surface normal) and a fraction of the $800 \mathrm{~nm}$ output, send into an etalon to generate a narrow-band visible laser pulse (s-polarized, $20 \mu \mathrm{J}, 810 \mathrm{~nm}$, angle $70^{\circ}$ ). The SFG signal with a central frequency of $674 \mathrm{~nm}$ is sent into a spectrometer and detected using an Andor Newton electron-multiplied charged coupled device (EMCCD) camera. The integration time of a single spectrum is between 100 and $200 \mathrm{~s}$ depending on the specific signal intensity and laser power. To correct for the frequency content of the IR beam, all SFG spectra are normalized to the non-resonant SFG response of $z$-cut quartz.

The salts are SigmaAldrich ACS grade with a purity of more than $99.9 \%$ and prior to dissolving them in $\mathrm{D}_{2} \mathrm{O}$, the salts are baked for $10 \mathrm{~h}$ at $650{ }^{\circ} \mathrm{C}$. No $\mathrm{CH}$ contamination could be observed in the SFG experiments. Both the $\mathrm{Na}_{2} \mathrm{SO}_{4}$ and $\mathrm{Na}_{2} \mathrm{CO}_{3}$ solutions prepared at room temperature had a molality of $1.8 \mathrm{~mol} / \mathrm{kg}$; the exact concentrations can be found in Table 1.

\section{Table 1. Used Salt Concentrations}

\begin{tabular}{cccc} 
salt & amount $/ \mathrm{g}$ & $\mathrm{D}_{2} \mathrm{O} / \mathrm{mL}$ & molality $/(\mathrm{mol} / \mathrm{kg})$ \\
$\mathrm{Na}_{2} \mathrm{CO}_{3}$ & $64 \pm 1$ & $300 \pm 1$ & $1.8 \pm 0.1$ \\
$\mathrm{Na}_{2} \mathrm{SO}_{4}$ & $62 \pm 1$ & $220 \pm 1$ & $1.8 \pm 0.1$ \\
\hline
\end{tabular}

To avoid heating effects from the laser, the sample is rotated such that subsequent laser pulses hit different spots. A chiller is used to keep the temperature of the sample constant during the measurement. To minimize evaporation, the temperature is maintained between 10 and $15^{\circ} \mathrm{C}$, and an automated syringe is used to keep the water level at a constant height throughout the measurement.

The phase-resolved spectra are recorded in a setup described in ref 18 .
To generate the narrowband pump (p-polarized, $\approx 90 \mathrm{~cm}^{-1}$ full width at half-maximum, fwhm, $55^{\circ}$ angle) at $\approx 2000 \mathrm{~nm}$, the idler output of a second Topas-c is sent into a BBO crystal to generate $\approx 1000 \mathrm{~nm}$ IR pulses. These pulses are overlapped with $800 \mathrm{~nm}$ pulses within a $\mathrm{LiNbO}_{3}$ crystal to generate the narrowband pump pulses. By tuning the frequency of the doubled idler and adjusting the phase-matching condition at the $\mathrm{LiNbO}_{3}$ crystal, the infrared pump laser can be tuned between 2350 and $2750 \mathrm{~cm}^{-1}$. The power varies from 5 to 10 $\mu \mathrm{J}$ depending on the central pump frequency. The crosscorrelation of pump and probe is emitted at about $570 \mathrm{~nm}$ after up-conversion with the visible laser pulse. Prior to each pump-probe experiment, the pump-probe delay dependency is measured and defines the instrument response function. The instrument response function typically has a temporal width of about 200 fs (Figure S1). To record pump-probe spectra, a chopper blocks every second laser pulse of the pump and a vibrating mirror separates the pumped and unpumped response sending them onto different sections of the CCD camera. By dividing the pumped and unpumped spectrum, one obtains the bleach:

$$
r_{S F G}(t, \omega)=I_{\text {pumped }}(t, \omega) / I_{\text {unpumped }}(\omega)
$$

Dividing the two intensities allows for the correction of correlated laser-induced uncertainties like spatial, spectral, and temporal drift. To follow the bleach in time, we use an automated delay stage to change the relative time difference between pump- and probe laser pulses with a minimum step size of $50 \mathrm{fs}$. The total time range is -10 to $+20 \mathrm{ps}$ where the pump arrives after (before) the probe for negative (positive) times. In total, 31 different pump-probe time delays were recorded.

To quantify the bleach intensity as a function of delay time, we average a region of $\pm 30 \mathrm{~cm}^{-1}$ around the central excitation frequency for each delay time. Here, $\pm 30 \mathrm{~cm}^{-1}$ equals $\pm \sigma$ of the pump pulse width in frequency space and allows one to extract the time-dependent trace:

$$
R_{S F G}(t)=\frac{1}{N_{\omega}} \sum_{\omega=-30 \mathrm{~cm}^{-1}}^{30 \mathrm{~cm}^{-1}} r_{S F G}(t, \omega)
$$

with $N_{\omega}$ being the number of measured probe frequencies, e.g., the number of pixels. To interpret the temporal dependency of $R_{S F G}(t)$, we use a kinetic model, explained in the following.

The time dependence of the bleach signal $R_{S F G}(t)$ provides insights into the vibrational dynamics at the water-air interface. In analogy to bulk experiments, energy relaxation at the water-air interface is commonly modeled with a fourlevel model depicted in Figure 1. In this model, a pump pulse excites a fraction of the molecules from the vibrational ground

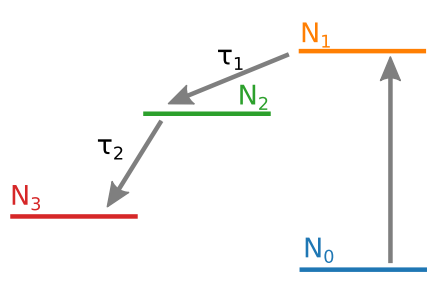

Figure 1. Energy level of the involved vibrational states. A pump pulse excites molecules from the ground level $N_{0}$ into $N_{1}$. After a lifetime of $\tau_{1}$ the molecules decay into an intermediate state $N_{2}$ and after $\tau_{2}$ into a long-lived heated ground state $N_{3}$. 
state $N_{0}$ into an excited state $N_{1}$. The excited molecules stay in $N_{1}$ for an average lifetime of $\tau_{1}$ and decay via an intermediate state $N_{2}$ into the heated ground state $N_{3}$. Due to the absorption of the laser beam, the temperature of the system changes. In particular, the cooling of the system occurs at time scales much longer than our experiment resolves. Thus a stable heated ground state is a reasonable assumption to account for these temperature effects. This kinetic model was first developed for pump-probe IR on bulk water and has been successfully used to describe the processes at the interface of water as well. ${ }^{19-21}$

The model in Figure 1 can be described with a system of coupled first-order linear differential equations given in eq 3-eq 6. $N$ is the total number of oscillators, $N_{i}(t)$ with $i \in\{0$, $1,2,3\}$ denotes the number of oscillators at time $t$ in state $i$ and $\tau_{i}$ is the corresponding lifetime of the state $N_{i}$.

$$
\begin{aligned}
& N_{0}(t)=N-N_{1}(t)-N_{2}(t)-N_{3}(t) \\
& \mathrm{d} N_{1}(t) / \mathrm{d} t=-N_{1}(t) / \tau_{1} \\
& \mathrm{~d} N_{2}(t) / \mathrm{d} t=N_{1}(t) / \tau_{1}-N_{2}(t) / \tau_{2} \\
& \mathrm{~d} N_{3}(t) / \mathrm{d} t=N_{2}(t) / \tau_{2}
\end{aligned}
$$

To the best of our knowledge, this model has always been solved numerically if used to describe SFG data. Typically a Gaussian-shaped excitation function is applied within eqs 4 and 5 to account for the temporal width of the excitation pulse. This makes it impossible to solve the differential equation analytically. However, as we show below, if one first solves the differential equations and then convolves this solution with a Gaussian instrument response function, a purely analytical model function can be derived. This has the practical advantage that no numerical differential equation solver is needed anymore upon fitting the data, leading to more robust fitting results.

The analytical solution to eq $3-6$ is given by the eqs $7-10$.

$$
\begin{aligned}
N_{0}(t)= & N-N_{1}(t)-N_{2}(t)-N_{3}(t) \\
N_{1}(t)= & N_{1}(0) \mathrm{e}^{-t / \tau_{1}} \\
N_{2}(t)= & \frac{1}{\tau_{1} / \tau_{2}-1}\left(\mathrm{e}^{-t / \tau_{1}}-\mathrm{e}^{-t / \tau_{2}}\right) N_{1}(0)+\mathrm{e}^{-t / \tau_{2}} N_{2}(0) \\
N_{3}(t)= & \left(\frac{1-\mathrm{e}^{-t / \tau_{1}}}{1-\tau_{2} / \tau_{1}}+\frac{\mathrm{e}^{-t / \tau_{2}}-1}{\tau_{1} / \tau_{2}-1}\right) N_{0}(0) \\
& +\left(1-\mathrm{e}^{-t / \tau_{2}}\right) N_{2}(0)+N_{3}(0)
\end{aligned}
$$

The pumping due to the probe is negligible and therefore we assume $N_{0}(0)=N-n, N_{1}(0)=n, N_{2}(0)=0$ and $N_{3}(0)=0$ at initial time $t=0$ with $n$ the number of initially excited oscillators. This simplifies eq 9 and eq 10 because only their first terms deviate from 0 . Assuming an instantaneous excitation pulse, the time dependent bleach $R_{S F G}(t)$ is modeled by the population of involved vibrational states as show in eq 11 .

$$
\begin{aligned}
R_{S F G}(t) & \propto S\left(t, \tau_{1}, \tau_{2}, c\right) \\
& =\left(N_{0}(t)-N_{1}(t)+N_{2}(t)+c N_{3}(t)\right)^{2} / N_{0}(0)^{2}
\end{aligned}
$$

This assumes the same spectral shape of the ground state $N_{0}(t)$ and the intermediate state $N_{2}(t)$ while $R_{S F G}$ is reduced by the amount of oscillators in the first excited state $N_{1}(t)$. The scaling factor $c$ accounts for the differences in the spectral shape of the heated ground state $N_{3}(t)$ in comparison with $N_{0}(t)$.

To account for the finite temporal width of the pump laser, eq 11 must be convolved with the temporal profile of the excitation pulse resulting in eq 12 .

$$
R_{S F G}(t) \propto S\left(t, \tau_{1}, \tau_{2}, c\right) * P_{e x t}(t)
$$

Here $P_{\text {ext }}(t)$ denotes an arbitrary shaped excitation function and $*$ a convolution. By assuming a Gaussian excitation profile with width $\sigma$ at time $\mu$ and amplitude $A$, the convolution integral of eq 12 can be solved analytically resulting in eq $13 .^{58}$

$$
\begin{aligned}
R_{S F G}(t) & \propto S^{\prime}\left(t, \tau_{1}, \tau_{2}, c, A, \sigma, \mu\right) \\
& =S\left(t, \tau_{1}, \tau_{2}, c\right) * G(t, A, \sigma, \mu)
\end{aligned}
$$

Out of the six parameters of eq 13 , three can be readily determined independently. The width $\sigma$ and the temporal position $\mu$ of the excitation beam can be deduced from the measured cross-correlation of the three involved laser beams (Figure S1). Previous results from pump-probe IR measurements for bulk $\mathrm{D}_{2} \mathrm{O}$ have indicated that $\tau_{2}$ is $700 \mathrm{fs} .{ }^{15,20,22}$ The free parameters are thus: The lifetime of the first excited state $\tau_{1}$, the amplitude of the excitation $A$ and the amplitude of the heat $c . S^{\prime}\left(t, \tau_{1}, c, A\right)$ is then used as a model function for a leastsquare fit to the measured $R_{S F G}(t)$ traces, and $\tau_{1}$ is thus extracted as the lifetime of the initially excited vibrational state.

\section{RESULTS AND DISCUSSION}

To investigate the impact of ions on the structure and the vibrational dynamics of the water-air interface, we first turn toward the structure. Structural information can be extracted from the interfacial vibrational spectrum, as stronger hydrogen bonds result in lower vibrational frequencies, and weaker hydrogen bonds in higher vibrational frequencies. ${ }^{15}$ The spectrum of just the interface is obtained by SFG spectroscopy in which the incoming infrared beam is in resonance with the molecular vibration of interest. ${ }^{14}$

An SFG spectrum of the $\mathrm{O}-\mathrm{D}$ stretch vibration of $\mathrm{D}_{2} \mathrm{O}$ is shown in Figure 2a. We use $\mathrm{D}_{2} \mathrm{O}$ instead of $\mathrm{H}_{2} \mathrm{O}$ as the vibrational dynamics is slower. ${ }^{24}$ This also means that conclusions drawn from $\mathrm{D}_{2} \mathrm{O}$ could be different for $\mathrm{H}_{2} \mathrm{O}$. Around $2750 \mathrm{~cm}^{-1}$ the free $\mathrm{O}-\mathrm{D}$ peak is visible. This relative sharp peak originates from $\mathrm{O}-\mathrm{D}$ groups pointing into the vapor phase and thus having no hydrogen bond partner. ${ }^{25}$ Due to the absence of hydrogen bond acceptors, these bonds vibrate at a relatively high frequency. The relatively broad feature from 2200 to $2550 \mathrm{~cm}^{-1}$ originates from hydrogenbonded $\mathrm{O}-\mathrm{D}$ vibrations and consists of two peaks centered around 2380 and $2510 \mathrm{~cm}^{-1}$. The origin of these two features has previously been investigated using different isotopic dilutions of $\mathrm{H}_{2} \mathrm{O}, \mathrm{D}_{2} \mathrm{O}$, methanol, and ethanol mixtures. This has allowed separating intramolecular and intermolecular contributions and led to the conclusion, that the intramolecular coupling generates the double-peak feature, while the intermolecular coupling induces a redshift of the $\mathrm{O}-\mathrm{H}$ stretch vibration. ${ }^{25-29}$

A solution of $1.8 \mathrm{M} \mathrm{Na}_{2} \mathrm{CO}_{3}$ in $\mathrm{D}_{2} \mathrm{O}$ shows a static SFG spectrum that is substantially different from that of the clean water surface. The strongly hydrogen-bonded region from 


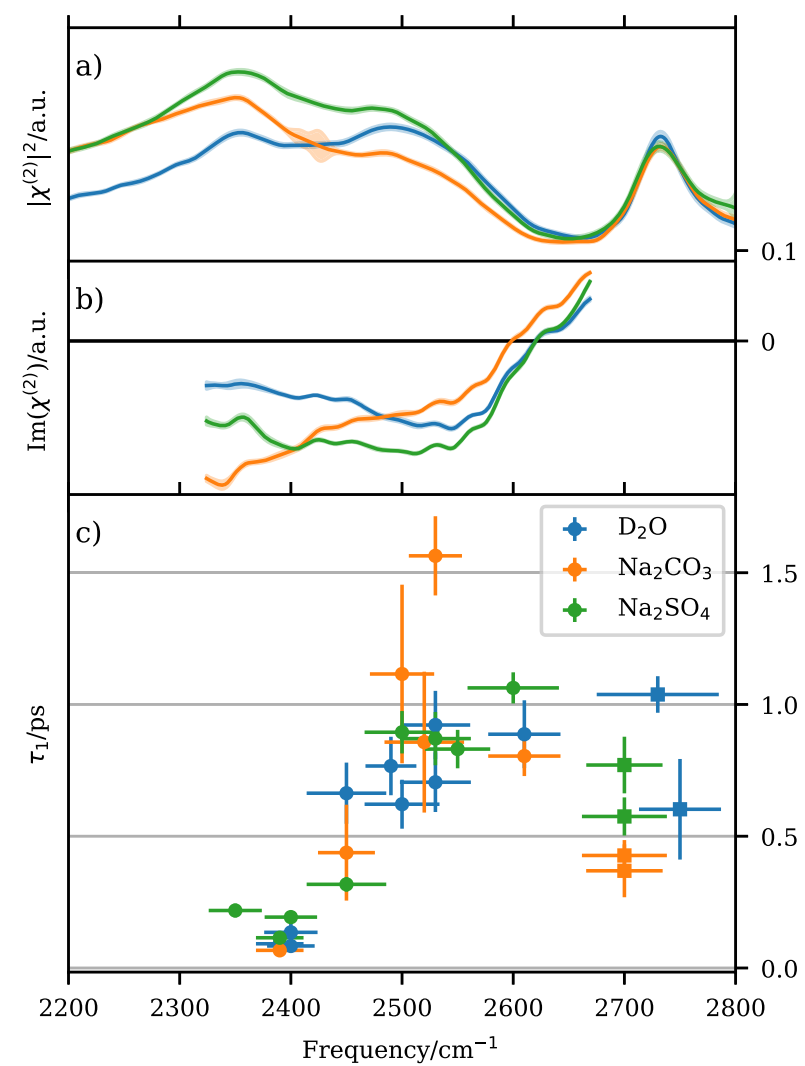

Figure 2. Conventional (a) and phase-resolved (b) SFG of O-D Stretch vibrations for $\mathrm{D}_{2} \mathrm{O}, 1.8 \mathrm{M} \mathrm{Na}_{2} \mathrm{SO}_{4}$ and $1.8 \mathrm{M} \mathrm{Na}_{2} \mathrm{CO}_{3}$. Shading around the lines indicates uncertainty of the data based on 10 averaged spectra. The region of enlarged uncertainty at $2400 \mathrm{~cm}^{-1}$ originates from an unusual cluster of cosmic muon induced spikes. ${ }^{23}$ (c) Lifetimes of the OD stretch vibration for the given pump and probe frequency respectively. Round dots indicate lifetimes extracted from a four-level model. Squares indicate lifetimes extracted from a single exponential fit. The $x$ error bars correspond to $1 \sigma$ excitation width, $y$ error bars are fit errors. In total, $\approx 24000$ SFG spectra were analyzed.

2200 to $2420 \mathrm{~cm}^{-1}$ is enhanced, whereas the weakly hydrogen bond region from 2420 to $2600 \mathrm{~cm}^{-1}$ is reduced in intensity. This hints toward an overall strengthening of the hydrogenbonded network in the presence of $\mathrm{Na}_{2} \mathrm{CO}_{3}$, while the free OD appears unchanged.

In the case of $\mathrm{Na}_{2} \mathrm{SO}_{4}$, the spectral changes are different. $\mathrm{A}$ concentration of $1.8 \mathrm{M} \mathrm{Na}_{2} \mathrm{SO}_{4}$ in $\mathrm{D}_{2} \mathrm{O}$ causes a signal enhancement from $2200 \mathrm{~cm}^{-1}$ on, until $2550 \mathrm{~cm}^{-1}$, while from this point on, until the end of the free OD peak at $2800 \mathrm{~cm}^{-1}$, the signal is identical with that of pure water. This can again be interpreted as a strengthening of the hydrogen bond network. $^{30,31}$

To investigate the orientation of the water molecules at the interface, we performed phase-resolved (PS) SFG measurements. Figure $2 b$ shows $\operatorname{Im}\left(\chi^{(2)}\right)$ of the same salt $-\mathrm{D}_{2} \mathrm{O}$ solutions. The hydrogen-bonded region of $\mathrm{D}_{2} \mathrm{O}$ around 2300 $\mathrm{cm}^{-1}$ to $2600 \mathrm{~cm}^{-1}$ has a negative $\operatorname{Im}\left(\chi^{(2)}\right)$, indicative of a net downward orientation of the $\mathrm{O}-\mathrm{D}$ transition dipole moment of interfacial water toward the bulk. ${ }^{32-34}$ Around $2620 \mathrm{~cm}^{-1}$, the $\operatorname{Im}\left(\chi^{(2)}\right)$ changes sign, showing that the free OD at around $2750 \mathrm{~cm}^{-1}$ has an opposite and thus net orientation with the $\mathrm{O}-\mathrm{D}$ transition dipole moment out of the interface.
Adding $1.8 \mathrm{M} \mathrm{Na}_{2} \mathrm{CO}_{3}$ reduces the $\operatorname{Im}\left(\chi^{(2)}\right)$ around the weakly bonded $2550 \mathrm{~cm}^{-1}$ region and increases the $\operatorname{Im}\left(\chi^{(2)}\right)$ below $2490 \mathrm{~cm}^{-1}$. This supports the hypothesis of $\mathrm{Na}_{2} \mathrm{CO}_{3}$, strengthening the interfacial hydrogen bonding network in comparison with the neat $\mathrm{D}_{2} \mathrm{O}$-air interface.

A solution of $1.8 \mathrm{M} \mathrm{Na}_{2} \mathrm{SO}_{4}$ in $\mathrm{D}_{2} \mathrm{O}$ enhances the $\operatorname{Im}\left(\chi^{(2)}\right)$ as well in both the strongly and weakly hydrogen-bonded region. The enhancement is similar as in the case of the static SFG spectrum from Figure 2a and can again be interpreted as a strengthening of the hydrogen-bonded network.

Nonetheless, this increase could also resemble $\chi^{(3)}$ contributions. From previous studies of charged interfaces, it is known that $\chi^{(3)}$ effects can play a role in the observed SFG and PS-SFG spectra. ${ }^{35-38}$ In particular, it was shown that the existence of a static electric field can induce an anisotropy throughout the bulk water and thus alter the SFG signal. It is commonly assumed that the electric field induced by the charge is screened by counterions effectively over the Debye length. The Debye length of saltwater with $2 \mathrm{M}$ ion concentrations is below $2 \AA$ and thus smaller than a single water layer, implying that the $\chi^{(3)}$ contributions should be insignificant. ${ }^{35,39,40}$ On the other hand, there is a remarkable agreement between the $\mathrm{Na}_{2} \mathrm{SO}_{4}$ PS-SFG spectrum and a constructed PS-SFG spectrum where the PS-SFG spectrum of neat $\mathrm{D}_{2} \mathrm{O}$ and $\chi^{(3)}$ as reported by Shen et al. ${ }^{36}$ is used see Figure S2. The resulting negative sign of the $\chi^{(3)}$ contribution implies that the positive ion $\mathrm{Na}^{+}$is closer to the surface than the negative ion $\mathrm{SO}_{4}{ }^{2-} \cdot{ }^{36}$ As $\mathrm{Na}^{+}$prefers bulk hydration, ${ }^{41}$ we conclude that both ions $\mathrm{Na}^{+}$and $\mathrm{SO}_{4}{ }^{2-}$ are sub surface. This is in line with simulation studies ${ }^{42}$ In the case of $\mathrm{Na}_{2} \mathrm{CO}_{3}$ the $\chi^{(3)}$ contribution to the signal could be excluded based on the spectral shape of the signal. For further information see Figure $\mathrm{S} 2$. We can only speculate about the reason for the difference of $\mathrm{Na}_{2} \mathrm{SO}_{4}$ and $\mathrm{Na}_{2} \mathrm{CO}_{3}$ in $\chi^{(3)}$ signal. It could be that $\mathrm{Na}_{2} \mathrm{SO}_{4}$ forms a double-layer-like structure, while $\mathrm{CO}_{3}{ }^{2-}$ and $\mathrm{Na}^{+}$ distribute more within the same water slab, creating an inplane electric field that is not probed by SFG.

Static and phase-resolved SFG spectra of $1 \mathrm{M} \mathrm{Na}_{2} \mathrm{SO}_{4}$ and 1 $\mathrm{M} \mathrm{Na}_{2} \mathrm{CO}_{3}$ in $\mathrm{H}_{2} \mathrm{O}$ have previously been reported by Allen and co-workers. ${ }^{8,9}$ Even though the overall spectral shapes between theirs and ours are comparable, there are some notable differences. At present it remains unclear whether these differences are the result of different concentrations, $\mathrm{D}_{2} \mathrm{O}$ vs $\mathrm{H}_{2} \mathrm{O}$ effects, impurities, or phase errors. ${ }^{43}$

From these and prior measurements, ${ }^{7-9}$ we conclude, that ions in bulk have a direct effect on the interfacial hydrogen bonded network. However, from the SFG and the PS SFG spectra nothing can be inferred about the vibrational dynamics at the interface. Do ions increase or decrease the lifetimes of the OD stretch vibration? Is the energy flow dynamics affected? To shed some light on these questions, we now turn toward the vibrational dynamics of the stretch vibration of the interfacial hydrogen bond network.

To probe the vibrational dynamics at the interface, we use pump-probe (PP) SFG. By tuning both, the pump-probe time delay and the central pump frequency, we obtain information about the relaxation time, as a function of the excitation frequency. ${ }^{44,45}$ Exemplary data of the ratio of pumped and unpumped SFG response (bleach) can be found in Figure S3. The traces shown in Figure 3 are constructed according to eq 2 . For each trace between 5 and 25 , transient SFG spectra at 31 different pump-probe time delays were measured and averaged. In Figure 3, traces for 


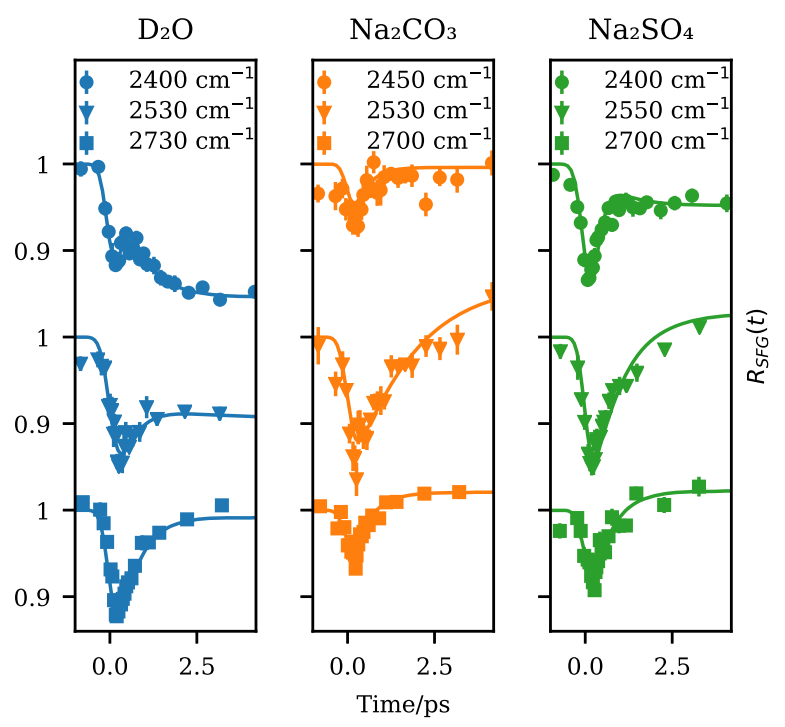

Figure 3. Traces of $\mathrm{D}_{2} \mathrm{O}, \mathrm{Na}_{2} \mathrm{CO}_{3}$, and $\mathrm{Na}_{2} \mathrm{SO}_{4}$ at three different pump frequencies. Lifetimes are extracted by fitting a four-level model. For pump frequencies greater than $2700 \mathrm{~cm}^{-1}$ a single exponential fit is used. Variations of $50 \mathrm{~cm}^{-1}$ in pump frequency can arise due to precise calibrations performed after the individual experiments. The shown traces were initially measured with the intention of having the same pump frequency.

$\mathrm{D}_{2} \mathrm{O}, \mathrm{Na}_{2} \mathrm{SO}_{4}$, and $\mathrm{Na}_{2} \mathrm{CO}_{3}$ at three different mean pump frequencies each, are shown. At negative times, the pump pulse arrives after the probe pulse, and thus, $R_{S F G}$ is 1 . At around $\mathrm{t}=$ $0 \mathrm{ps}$, the pump and the probe pulses start to overlap in time, and the ground state of the sample is bleached, resulting in a decrease of $R_{S F G}$ and a consecutive recovery of the signal.

One notes that the magnitude of $R_{S F G}$ depends on the power of the pump pulse and the IR absorption cross-section at the pumped frequency. Because the pump power is highest at around $2500 \mathrm{~cm}^{-1}$ as is the IR absorption, the largest bleach can be seen around this pump frequency (see Figure S4). Remarkably, $\mathrm{D}_{2} \mathrm{O}$ pumped close to the free OD at $2730 \mathrm{~cm}^{-1}$ is an exception to that rule, as we observe a relative high $R_{S F G}$ of $10 \%$. Taking the overall picture into account, one can see that the amplitude is largest for $\mathrm{Na}_{2} \mathrm{SO}_{4}$ and smallest for $\mathrm{Na}_{2} \mathrm{CO}_{3}$ with $\mathrm{D}_{2} \mathrm{O}$ in between.

For long delay times $(t \geq 4 \mathrm{ps}) R_{S F G}$ of Figure 3 converges to a value different from one, but constant on the picosecond time scale of the experiment. This late time signal represents, as discussed in detail in the Supporting Information, the elevated temperature of the system and the subsequent weakening of the $\mathrm{H}$-bonds after vibrational relaxation. Depending on the influence of the heat, the value of this constant can be either smaller or larger than one. $\mathrm{D}_{2} \mathrm{O}$ pumped at $2400 \mathrm{~cm}^{-1}$ is an example for a long time signal smaller than one, while most others show a signal exceeding one.

To extract relaxation times, the traces are modeled (see Figure 3). The reproducible bump in the trace of $\mathrm{D}_{2} \mathrm{O}$ at delay times of a few hundred femtoseconds, pumped at $2400 \mathrm{~cm}^{-1}$, shows that a model with at least two lifetimes is needed to describe the process. As such we use the four-level model previously described in Figure 1 for pump frequencies exciting within the range of the bonded OD stretch vibration (pump frequency $\left.<2700 \mathrm{~cm}^{-1}\right)$. For the free OD region $\left(>2700 \mathrm{~cm}^{-1}\right)$ a single exponential model is used. ${ }^{46-48}$ The extracted lifetimes of the first excited state $\tau_{1}$ for all measured pump frequencies and samples are shown in Figure 2 (c).

Figure $2 \mathrm{c}$ shows that we observe the shortest lifetimes of $\approx 0.2$ ps for all samples around a pump frequencies of 2400 $\mathrm{cm}^{-1}$, and Figure S1 makes clear that the instrument response function is the limiting property. Upon increasing the excitation frequency to $2600 \mathrm{~cm}^{-1}$ an increase in lifetime up to almost 1 ps is found. In the case of $\mathrm{D}_{2} \mathrm{O}$, this nicely matches previously obtained results. ${ }^{16}$ It was hypothesized that the reason for this heterogeneity is the coupling of the OD-stretch mode with an overtone of the OD bending mode. ${ }^{49-52}$ Around $2400 \mathrm{~cm}^{-1}$, the first overtone of the bending mode overlaps with the OD-Stretch vibration. Therefore the overtone provides an efficient relaxation path to the excited molecules. Upon exciting the surface at a frequency of $2500 \mathrm{~cm}^{-1}$ or higher, the coupling to the overtone of the bending mode becomes less efficient, leading to slower vibrational relaxation. An alternative explanation is provided from theoretical work, interpreting this frequency dependency as a hydration layer effect and correlating it with the number of $\mathrm{H}$-bonds per water molecules. 53

Pumping close to the free OD peak $\left(2700-2750 \mathrm{~cm}^{-1}\right)$ shows lifetimes around $0.5 \mathrm{ps}$ for the stretch vibration. With $\mathrm{Na}_{2} \mathrm{SO}_{4}$ and $\mathrm{D}_{2} \mathrm{O}$ having roughly 700 fs lifetime and $\mathrm{Na}_{2} \mathrm{CO}_{3}$ 400 fs. The free OD was previously studied and showed lifetimes around 800 fs. $^{48}$

As apparent from Figure $2 c$, the difference in lifetimes between the three samples $\mathrm{D}_{2} \mathrm{O}, \mathrm{Na}_{2} \mathrm{CO}_{3}$, and $\mathrm{Na}_{2} \mathrm{SO}_{4}$ are minimal. For specific pump frequencies, the lifetime of the samples can be different by more than $2 \sigma$, but the error bars capture only the stability of the individual experiment, and do not account for correlations between the fitting parameters and sample-to-sample variations. Taking the complete picture into account there is no clear difference between the three samples. This is somewhat surprising, because, as can be seen in Figure 2 , parts a and $b$, the static SFG spectra of $\mathrm{Na}_{2} \mathrm{SO}_{4}$ and $\mathrm{Na}_{2} \mathrm{CO}_{3}$ show significant differences from $\mathrm{D}_{2} \mathrm{O}$, hinting toward structural changes induced by the $\mathrm{CO}_{3}{ }^{2-}$ and $\mathrm{SO}_{4}{ }^{2-}$ ions. Nevertheless, this structural change apparently does not significantly influence the vibrational dynamics of the involved vibrational state within the uncertainty of the experiment. It could, however, be that larger and more granular sampling rate of the pump frequency, or an improved signal-to-noise ratio, reveals differences between $\mathrm{D}_{2} \mathrm{O}-\mathrm{D}_{2} \mathrm{O}$ and $\mathrm{D}_{2} \mathrm{O}$-ion bindings. However, given the fact that differences in the static spectra appear through bands of about $100 \mathrm{~cm}^{-1}$, a $60 \mathrm{~cm}^{-1}$ wide pump pulse should be sufficient to resolve these differences. Furthermore, lifetimes with differences of less than $0.2 \mathrm{ps}$ are challenging to detect with the currently available measurement techniques.

\section{CONCLUSION}

Returning to the questions presented in the introduction of this manuscript, we conclude that the interfacial water structure of aerosol particles is ion-dependent. As such, the surface structure can be responsible for unique properties of aerosol particles. Still, because the interfacial vibrational dynamics seem to be unaffected by the ion content, models of excess energy transport do not need to take the specific ion content into account. Instead, models taking solely the properties of the static $\mathrm{H}$-bond network of water into account are likely sufficient to describe the details of excess energy transport accurately, even for aerosol particles with high ion 
concentrations. We further note that the four-level model, first developed for bulk water, can be used for interfacial water as well, even if the H-bond network is perturbed by high ionic concentrations. In addition, we show that the lifetime of the stretch vibration depends on the excitation frequency and ranges from about 0.2 ps to over 1 ps.

\section{ASSOCIATED CONTENT}

\section{S Supporting Information}

The Supporting Information is available free of charge on the ACS Publications website at DOI: 10.1021/acs.jpcb.9b08131.

Measurement of time resolution, $\chi^{(3)}$ Induced SFG response, bleach spectra, IR absorption spectra, spectral heat effects (PDF)

\section{AUTHOR INFORMATION}

\section{Corresponding Author}

*(E.H.G.B.) E-mail: backus@mpip-mainz.mpg.de.

\section{ORCID}

Mischa Bonn: 0000-0001-6851-8453

Ellen H. G. Backus: 0000-0002-6202-0280

\section{Notes}

The authors declare no competing financial interest.

\section{ACKNOWLEDGMENTS}

The authors thank G. Florian and M. van Zadel for engineering, $H$. Menges for laser maintenance, J. Worm for programming and S. Das, S. Pezzotti, D. Galimberti, M-P. Gaigeot, and M. Grechko for fruitful discussions. This work is funded by the Deutsche Forschungsgemeinschaft (DFG, German Research Foundation) - BA 5008/3. This research made use of the MINUIT algorithm ${ }^{54}$ via the iminuit ${ }^{55}$ Python interface, Matplotlib, ${ }^{56}$ and Numpy. ${ }^{57}$

\section{REFERENCES}

(1) Bauer, S. E.; Balkanski, Y.; Schulz, M.; Hauglustaine, D. A.; Dentener, F. Global Modeling of Heterogeneous Chemistry on Mineral Aerosol Surfaces: Influence on Tropospheric Ozone Chemistry and Comparison to Observations. J. Geophys. Res. 2004, 109, 2003JD003868.

(2) Andreae, M. O.; Crutzen, P. J. Atmospheric Aerosols: Biogeochemical Sources and Role in Atmospheric Chemistry. Science 1997, 276, 1052-1058.

(3) Andreae, M. O.; Merlet, P. Emission of Trace Gases and Aerosols From Biomass Burning. Global Biogeochemical Cycles 2001, $15,955-966$.

(4) Ramanathan, V.; Crutzen, P. J.; Kiehl, J. T.; Rosenfeld, D. Aerosols, Climate, and the Hydrological Cycle. Science 2001, 294, 2119-2124.

(5) Wang, Y.; Zhuang, G.; Tang, A.; Yuan, H.; Sun, Y.; Chen, S.; Zheng, A. The Ion Chemistry and the Source of $\mathrm{PM}_{2.5}$ Aerosol in Beijing. Atmos. Environ. 2005, 39, 3771-3784.

(6) Field, C. B.; Raupach, M. R. The Global Carbon Cycle: Integrating Humans, Climate, and the Natural World; Island Press: 2004; Vol. 62.

(7) Hua, W.; Verreault, D.; Allen, H. C. Relative Order of Sulfuric Acid, Bisulfate, Hydronium, and Cations at the Air-Water Interface. J. Am. Chem. Soc. 2015, 137, 13920-13926.

(8) Hua, W.; Chen, X.; Allen, H. C. Phase-Sensitive Sum Frequency Revealing Accommodation of Bicarbonate Ions, and Charge Separation of Sodium and Carbonate Ions Within the Air/Water Interface. J. Phys. Chem. A 2011, 115, 6233-6238.

(9) Hua, W.; Jubb, A. M.; Allen, H. C. Electric Field Reversal of $\mathrm{Na}_{2} \mathrm{SO}_{4},\left(\mathrm{NH}_{4}\right)_{2} \mathrm{SO}_{4}$, and $\mathrm{Na}_{2} \mathrm{CO}_{3}$ Relative to $\mathrm{aCaCl}_{2} \mathrm{nd} \mathrm{NaCl}$ at the
Air/Aqueous Interface Revealed by Heterodyne Detected PhaseSensitive Sum Frequency. J. Phys. Chem. Lett. 2011, 2, 2515-2520.

(10) Vogler, E. A. Structure and Reactivity of Water at Biomaterial Surfaces. Adv. Colloid Interface Sci. 1998, 74, 69-117.

(11) Jungwirth, P.; Tobias, D. J. Ions at the Air/Water Interface. J. Phys. Chem. B 2002, 106, 6361-6373.

(12) Knipping, E. M.; Lakin, M. J.; Foster, K. L.; Jungwirth, P.; Tobias, D. J.; Gerber, R. B.; Dabdub, D.; Finlayson-Pitts, B. J. Experiments and Simulations of Ion-Enhanced Interfacial Chemistry on Aqueous $\mathrm{NaCl}$ Aerosols. Science 2000, 288, 301-306.

(13) Ataka, K.-i.; Yotsuyanagi, T.; Osawa, M. Potential-Dependent Reorientation of Water Molecules at an Electrode/Electrolyte Interface Studied by Surface-Enhanced Infrared Absorption Spectroscopy. J. Phys. Chem. 1996, 100, 10664-10672.

(14) Lambert, A. G.; Davies, P. B.; Neivandt, D. J. Implementing the Theory of Sum Frequency Generation Vibrational Spectroscopy: A Tutorial Review. Appl. Spectrosc. Rev. 2005, 40, 103-145.

(15) Rey, R.; Møller, K. B.; Hynes, J. T. Hydrogen Bond Dynamics in Water and Ultrafast Infrared Spectroscopy. J. Phys. Chem. A 2002, 106, 11993-11996.

(16) van der Post, S. T.; Hsieh, C.-S.; Okuno, M.; Nagata, Y.; Bakker, H. J.; Bonn, M.; Hunger, J. Strong Frequency Dependence of Vibrational Relaxation in Bulk and Surface Water Reveals SubPicosecond Structural Heterogeneity. Nat. Commun. 2015, 6, 8384.

(17) Livingstone, R. A.; Nagata, Y.; Bonn, M.; Backus, E. H. G. Two Types of Water at the Water-Surfactant Interface Revealed by TimeResolved Vibrational Spectroscopy. J. Am. Chem. Soc. 2015, 137, 14912-14919.

(18) Khatib, R.; Backus, E. H. G.; Bonn, M.; Perez-Haro, M.-J.; Gaigeot, M.-P.; Sulpizi, M. Water Orientation and Hydrogen-Bond Structure at the Fluorite/Water Interface. Sci. Rep. 2016, 6, 24287.

(19) Steinel, T.; Asbury, J. B.; Zheng, J.; Fayer, M. D. Watching Hydrogen Bonds Break: A Transient Absorption Study of Water. J. Phys. Chem. A 2004, 108, 10957-10964.

(20) McGuire, J. A.; Shen, Y. R. Ultrafast Vibrational Dynamics at Water Interfaces. Science 2006, 313, 1945-1948.

(21) Livingstone, R. A.; Zhang, Z.; Piatkowski, L.; Bakker, H. J.; Hunger, J.; Bonn, M.; Backus, E. H. G. Water in Contact with a Cationic Lipid Exhibits Bulklike Vibrational Dynamics. J. Phys. Chem. B 2016, 120, 10069-10078.

(22) Loparo, J. J.; Roberts, S. T.; Tokmakoff, A. Multidimensional Infrared Spectroscopy of Water. I. Vibrational Dynamics in TwoDimensional IR Line Shapes. J. Chem. Phys. 2006, 125, 194521.

(23) Leach, R. W.; Gursky, H. The Cosmic Ray Background in Charge Coupled Devices. Publ. Astron. Soc. Pac. 1979, 91, 855.

(24) Smits, M.; Ghosh, A.; Sterrer, M.; Müller, M.; Bonn, M. Ultrafast Vibrational Energy Transfer Between Surface and Bulk Water at the Air-Water Interface. Phys. Rev. Lett. 2007, 98, 098302.

(25) Du, Q.; Superfine, R.; Freysz, E.; Shen, Y. R. Vibrational Spectroscopy of Water at the Vapor/Water Interface. Phys. Rev. Lett. 1993, 70, 2313-2316.

(26) Sovago, M.; Campen, R. K.; Wurpel, G. W. H.; Müller, M.; Bakker, H. J.; Bonn, M. Vibrational Response of Hydrogen-Bonded Interfacial Water is Dominated by Intramolecular Coupling. Phys. Rev. Lett. 2008, 100, 173901.

(27) Nihonyanagi, S.; Ishiyama, T.; Lee, T.-k.; Yamaguchi, S.; Bonn, M.; Morita, A.; Tahara, T. Unified Molecular View of the Air/Water Interface Based on Experimental and Theoretical $\chi(2)$ Spectra of an Isotopically Diluted Water Surface. J. Am. Chem. Soc. 2011, 133, $16875-16880$.

(28) Schaefer, J.; Backus, E. H. G.; Nagata, Y.; Bonn, M. Both Interand Intramolecular Coupling of $\mathrm{O}-\mathrm{H}$ Groups Determine the Vibrational Response of the Water/Air Interface. J. Phys. Chem. Lett. 2016, 7, 4591-4595.

(29) Nagata, Y.; Mukamel, S. Vibrational Sum-Frequency Generation Spectroscopy at the Water/Lipid Interface: Molecular Dynamics Simulation Study. J. Am. Chem. Soc. 2010, 132, 6434-6442. 
(30) Gale, G. M.; Gallot, G.; Hache, F.; Lascoux, N.; Bratos, S.; Leicknam, J.-C. Femtosecond Dynamics of Hydrogen Bonds in Liquid Water: A Real Time Study. Phys. Rev. Lett. 1999, 82, 1068-1071.

(31) Sovago, M.; Kramer Campen, R.; Bakker, H. J.; Bonn, M. Hydrogen Bonding Strength of Interfacial Water Determined with Surface Sum-Frequency Generation. Chem. Phys. Lett. 2009, 470, 712.

(32) Superfine, R.; Huang, J. Y.; Shen, Y. R. Phase Measurement for Surface Infrared-Visible Sum-Frequency Generation. Opt. Lett. 1990, $15,1276-1278$

(33) Stiopkin, I. V.; Jayathilake, H. D.; Bordenyuk, A. N.; Benderskii, A. V. Heterodyne-Detected Vibrational Sum Frequency Generation Spectroscopy. J. Am. Chem. Soc. 2008, 130, 2271-2275.

(34) Yamaguchi, S. Development of Single-Channel HeterodyneDetected Sum Frequency Generation Spectroscopy and its Application to the Water/Vapor Interface. J. Chem. Phys. 2015, 143, 034202.

(35) Jena, K. C.; Covert, P. A.; Hore, D. K. The Effect of Salt on the Water Structure at a Charged Solid Surface: Differentiating Secondand Third-Order Nonlinear Contributions. J. Phys. Chem. Lett. 2011, 2, 1056-1061.

(36) Wen, Y.-C.; Zha, S.; Liu, X.; Yang, S.; Guo, P.; Shi, G.; Fang, H.; Shen, Y. R.; Tian, C. Unveiling Microscopic Structures of Charged Water Interfaces by Surface-Specific Vibrational Spectroscopy. Phys. Rev. Lett. 2016, 116, 016101.

(37) Schaefer, J.; Gonella, G.; Bonn, M.; Backus, E. H. G. SurfaceSpecific Vibrational Spectroscopy of the Water/Silica Interface: Screening and Interference. Phys. Chem. Chem. Phys. 2017, 19, $16875-16880$.

(38) Pezzotti, S.; Galimberti, D. R.; Shen, Y. R.; Gaigeot, M.-P. Structural Definition of the Bil and Dl: A New Universal Methodology to Rationalize Non-Linear $\chi^{(2)}(\omega)$ SFG Signals at Charged Interfaces, Including $\chi^{(3)}(\omega)$ Contributions. Phys. Chem. Chem. Phys. 2018, 20, 5190-5199.

(39) Gragson, D. E.; McCarty, B. M.; Richmond, G. L. Ordering of Interfacial Water Molecules at the Charged Air/Water Interface Observed by Vibrational Sum Frequency Generation. J. Am. Chem. Soc. 1997, 119, 6144-6152.

(40) Smith, A. M.; Lee, A. A.; Perkin, S. The Electrostatic Screening Length in Concentrated Electrolytes Increases with Concentration. J. Phys. Chem. Lett. 2016, 7, 2157-2163.

(41) Das, S.; Bonn, M.; Backus, E. H. G. The Surface Affinity of Cations Depends on Both the Cations and the Nature of the Surface. J. Chem. Phys. 2019, 150, 044706.

(42) Imamura, T.; Mizukoshi, Y.; Ishiyama, T.; Morita, A. Surface Structures of $\mathrm{NaF}$ and $\mathrm{Na}_{2} \mathrm{SO}_{4}$ Aqueous Solutions: Specific Effects of Hard Ions on Surface Vibrational Spectra. J. Phys. Chem. C 2012, 116, 11082-11090.

(43) Nihonyanagi, S.; Kusaka, R.; Inoue, K.-i.; Adhikari, A.; Yamaguchi, S.; Tahara, T. Accurate Determination of Complex $\chi^{(2)}$ Spectrum of the Air/Water Interface. J. Chem. Phys. 2015, 143, 124707.

(44) Bonn, M.; Ueba, H.; Wolf, M. Theory of Sum-Frequency Generation Spectroscopy of Adsorbed Molecules Using the Density Matrix Method-Broadband Bibrational Sum-Frequency Generation and Applications. J. Phys.: Condens. Matter 2005, 17, S201-S220.

(45) Hayashi, M.; Shiu, Y.-J.; Liang, K. K.; Lin, S. H.; Shen, Y. R. Theory of Time-Resolved Sum-Frequency Generation and Its Applications To Vibrational Dynamics of Water. J. Phys. Chem. A 2007, 111, 9062-9069.

(46) Xiao, S.; Figge, F.; Stirnemann, G.; Laage, D.; McGuire, J. A. Orientational Dynamics of Water at an Extended Hydrophobic Interface. J. Am. Chem. Soc. 2016, 138, 5551-5560.

(47) Hsieh, C.-S.; Campen, R. K.; Vila Verde, A. C.; Bolhuis, P.; Nienhuys, H.-K.; Bonn, M. Ultrafast Reorientation of Dangling OH Groups at the Air-Water Interface Using Femtosecond Vibrational Spectroscopy. Phys. Rev. Lett. 2011, 107, 116102.

(48) Zhang, Z.; Piatkowski, L.; Bakker, H. J.; Bonn, M. Ultrafast Vibrational Energy Transfer at the Water/Air Interface Revealed by
Two-Dimensional Surface Vibrational Spectroscopy. Nat. Chem. 2011, 3, 888-893.

(49) Imoto, S.; Xantheas, S. S.; Saito, S. Ultrafast Dynamics of Liquid Water: Energy Relaxation and Transfer Processes of the $\mathrm{OH}$ Stretch and the HOH Bend. J. Phys. Chem. B 2015, 119, 1106811078 .

(50) Rey, R.; Møller, K. B.; Hynes, J. T. Ultrafast Vibrational Population Dynamics of Water and Related Systems: A Theoretical Perspective. Chem. Rev. 2004, 104, 1915-1928.

(51) Rey, R.; Hynes, J. T. Vibrational Energy Relaxation of HOD in Liquid $\mathrm{D}_{2} \mathrm{O}$. J. Chem. Phys. 1996, 104, 2356-2368.

(52) Ashihara, S.; Huse, N.; Espagne, A.; Nibbering, E.; Elsaesser, T. Vibrational Couplings and Ultrafast Relaxation of the $\mathrm{O}-\mathrm{H}$ Bending Mode in Liquid. $\mathrm{H}_{2} \mathrm{O}$. Chem. Phys. Lett. 2006, 424, 66-70.

(53) Lesnicki, D.; Sulpizi, M. A Microscopic Interpretation of Pump-Probe Vibrational Spectroscopy Using Ab Initio Molecular Dynamics. J. Phys. Chem. B 2018, 122, 6604-6609.

(54) James, F.; Roos, M. Minuit - A System for Function Minimization and Analysis of the Parameter Errors and Correlations. Comput. Phys. Commun. 1975, 10, 343-367.

(55) iminuit team, Iminuit - A Python Interface to Minuit. https:// github.com/iminuit/iminuit. Accessed: 2019-02-28.

(56) Hunter, J. D. Matplotlib: A 2D Graphics Environment. Comput. Sci. Eng. 2007, 9, 90-95.

(57) Oliphant, T. NumPy: A Guide to NumPy; Trelgol Publishing: USA, 2006; http://www.numpy.org/. Accessed: 2019-02-28.

(58) A reference implementation of the function is at https://github. com/deisi/bleach-models. A python implementation is attached as ./four_level_model.py. Due to the associativity of the convolution under scalar multiplication, the same model can be used for difference based bleach, as a constant offset of -1 is the only difference. 